Original Research Paper

\title{
Controlling Chaos of the Ricker Population Model by OGY Method
}

\author{
Guo Feng \\ School of Data and Computer Science, Shandong Women's University, Jinan, China 250300, China
}

Article history

Received: 03-07-2020

Revised: 02-09-2020

Accepted: 07-09-2020

Email: sdwugf@163.com

\begin{abstract}
The chaotic dynamics of the Ricker mapping are studied. Controlling chaos of the Ricker population model is searched by OGY control method. The dynamic behavior in the Ricker mapping is very complex in different values of $a$. When the value of $a$ is changed from 0.455 to 90 , the mapping goes through doubling bifurcation to NeimarkSacker bifurcation. Several strange attractors coexist at last. Through the numerical simulation and analysis of the bifurcation and phase diagrams of the mapping, it is consistent with the theoretical analysis. Studies have shown that ecological balance can be achieved by appropriately adjusting the birth rate $a$, survival rate $b$.
\end{abstract}

Keywords: Ricker Mapping, Control Chaos Motion, OGY Method

\section{Introduction}

The Leslie model (Caswell, 2001) is an individual population model mainly used for key age structures of demographics and the conservation ecology.

Following ecologists' discoveries and in order to help ecologists model this kind of groups, ubiquitous age structure begins an extension study of the Leslie population model. in which the specific probability of survival and reproduction depends on population density. These nonlinear extensions usually have very complex dynamic behavior. Ugarcovici and Weiss $(2004 ; 2007)$ research the Ricker model. As the total population increases, the fertility rate declined monotonously and the fertility rate declined exponentially population size, the fertility decay is exponential. Ugarcovici and Weiss (2007) proved that for some parameter regions, the strange attractor of Ricker model has an unique physical probability measurements.

Ogata (1990) proposed a method of chaos control. Ott et al. (1990) have further improved this method. Because this method is suggested by Ott, Grebogi and Yorke and is named the OGY control method.

Many scholars according to the situation, various improvement measures are proposed and the OGY method has been developed a bit (Pyragas, 1993). It has shown that the energy-based feedback controller can change the chaotic dynamics into different chaotic dynamics (Reza Ahrabi and Kobravi, 2019). The parameter that linear feedback control to control the modified Automatic Van der Paul circuit (ADVP) (El-Sayed et al., 2013). Chaos in a memoristor based circuit and nonlinear circuits are controlled using the method of linear feedback control (Saini and Saini, 2014; Iu et al., 2011; Nik et al., 2015). Research on chaos control of 2-D of shock vibration system based on OGY method (Feng et al., 2018). Romeiras et al. (1992) through the use of polling mapping technology in system control, the OGY method has been further improved. Berdnikov and Lokhin (2019) discussed a nonstationary nonlinear system by the improved OGY method. Kocarev and Parlitz (1995) used general approach to control chaotic motion.

The chaotic dynamics of the Ricker mapping are investigated and the chaos is controlled. Analyze 1 control period and 2 unstable periods of the periodic trajectory. Whether you are living in a balanced state or growing up in chaos or demographic chaos. These studies can provide evidence and contribute to biological or ecological research.

In section three, the dynamic behavior of the twogeneration Ricker type model. In section four, pole placement technique for controlling chaos is introduced. In section five, I utilize the OGY control way to control chaos with unstable fixed points and we can get very short discussion from section six.

\section{The Leslie Model}

\section{The Linear Leslie Model}

Leslie $(1945 ; 1948)$ introduced a linear population model with the following age structure is introduced. Think about the population divided by age group or age group, we call it 1,2 and $d$ generations. Suppose that at 
time $\mathrm{t}$, the number of individuals in the kth age group (in households). People in the second generation $d$ are survivors of the previous generation at time $t$, so people assume 2, 3, d:

$n_{k}(t+1)=p_{k-1} n_{k-1}(t)$

where, $p_{i}, i=1, \ldots, p_{d^{-}} 1$ is the possibility that a person can survive a generation at the age of $i$. A new member of the one-year-old age group cannot be a survivor of another age group, but must come from reproduction. So Leslie believes that:

$n_{1}(t+1)=f_{1} n_{1}(t)+f_{2} n_{2}(t)+\cdots+f_{d} n_{d}(t)$

where, $f_{i}$ is the per capital fertility of generation $i$. Hence:

$$
\left(\begin{array}{c}
n_{1} \\
n_{2} \\
\vdots \\
n_{d}
\end{array}\right)(t+1)=\left(\begin{array}{ccccc}
f_{1} & f_{2} & \cdots & f_{d-1} & f_{d} \\
p_{1} & 0 & \cdots & 0 & 0 \\
\vdots & \vdots & \cdots & \vdots & \vdots \\
0 & 0 & \cdots & p_{d-1} & 0
\end{array}\right)\left(\begin{array}{c}
n_{1} \\
n_{2} \\
\vdots \\
n_{d}
\end{array}\right)(t)
$$

or more compactly:

$$
n(t+1)=\mathrm{A} n(t)
$$

Classified by age, this very special matrix A is called the Leslie matrix and we can see that Matrix A is not negative, it only has the first row and the diagonal pair of positive items. Demographers and demographers construct matrix A using life table analysis.

Most of the Leslie models used for real population predictions use 5 year old models instead of 3 generations. In this case matrix $A$ will be a $10 \times 10$ matrix. The Leslie model has been fully processed in Caswell (2001)'s "Matrix Total Model" book.

The Nonlinear Leslie Model

$$
\begin{gathered}
A(N)=\left(\begin{array}{ccccc}
f_{1} \mathrm{e}^{-\lambda N} & f_{2} \mathrm{e}^{-\lambda N} & \cdots & f_{d-1} \mathrm{e}^{-\lambda N} & f_{d} \mathrm{e}^{-\lambda N} \\
p_{1} & 0 & \cdots & 0 & 0 \\
\vdots & \vdots & \cdots & \vdots & \vdots \\
0 & 0 & \cdots & p_{d-1} & 0
\end{array}\right) \\
n_{1}(t+1)=\left(f_{1} n_{1}(t)+f_{2} n_{2}(t)+\cdots+f_{d} n_{d}(t)\right) \mathrm{e}^{-\lambda\left(n_{1}(t)+n_{2}(t)+\ldots n_{d}(t)\right)} \\
n_{2}(t+1)=p_{1} n_{1}(t) \\
\cdots \cdots \cdots \cdots \cdots \cdots \cdots \\
n_{d}(t+1)=p_{d-1} n_{d-1}(t)
\end{gathered}
$$

The associated $2 d$-parameter nonlinear dynamical system $T: R_{d} \rightarrow R_{d}$ is:

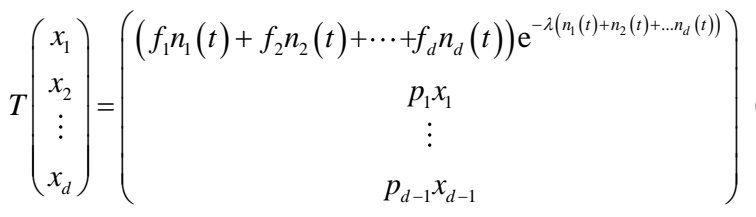

For two and three generations, the corresponding systems are:

$T(x, y)=\left(\left(f_{1} x+f_{2} y\right) \mathrm{e}^{-\lambda(x+y)}, p_{1} x\right)$

and:

$T(x, y, z)=\left(\left(f_{1} x+f_{2} y+f_{3} z\right) \mathrm{e}^{-\lambda(x+y+z)}, p_{1} x, p_{2} y\right)$

\section{The Dynamic Behavior of the Two- Generation Ricker Type Model}

The Ricker-type recruitment population model is described by the two dimensional mapping $R_{a, b}: \mathbb{R}_{+}^{2} \rightarrow \mathbb{R}_{+}^{2}:$

$R_{a, b}(x, y):\left\{\begin{array}{c}f(x, y)=(a x+\gamma a y) \mathrm{e}^{-\lambda(x+y)} \\ g(x, y)=b x\end{array}\right.$

In the $0 \mathrm{~h}$ group, $b$ is the survival rate from the first to second age group and $\lambda>0$. For the parameters $\gamma=0.2$, $\lambda=0.1$ and fixed survival rate $b=0.2$. While the first fertility rate has changed from 60-90, the dynamic behavior of the Ricker mapping exhibits complicated dynamical behavior. The bifurcation diagrams of Ricker mapping is shown in Fig. 1a-1d.

This type of bifurcation cannot occur for onedimensional mappings. $0.455<a<17.5$ (Fig. 1b). At $a=$ 17.5 , there is a saddle knot branch in cycle 2 creates a pull cycle orbits and its associated period- 2 orbit. At $a=$ 34.5, the stable fixed point undergoes a doubling bifurcation (Fig. 1c). For 34.5<a<90 (Fig. 1d, where there are couple different transfer attractors, each of which has a great appeal. At $a=85$, strange attractors coexist with a constant curve. This constant closed curve is split into a strange tractor.

The phase diagrams of the mapping when $a$ takes different values are consistent with the analysis of the above bifurcation diagrams (Fig. 2).

As shown in Fig. 3, if $0<a<85$, then the largest Lyapunov exponent is less than 0 . When $a>85$, the largest Lyapunov exponent is larger than 0 . The dynamic behavior is confusing. Then we can find the confusing part as LE $\max =22$. 
Guo Feng / American Journal of Biochemistry and Biotechnology 2020, 16 (3): 424.431 DOI: 10.3844/ajbbsp.2020.424.431

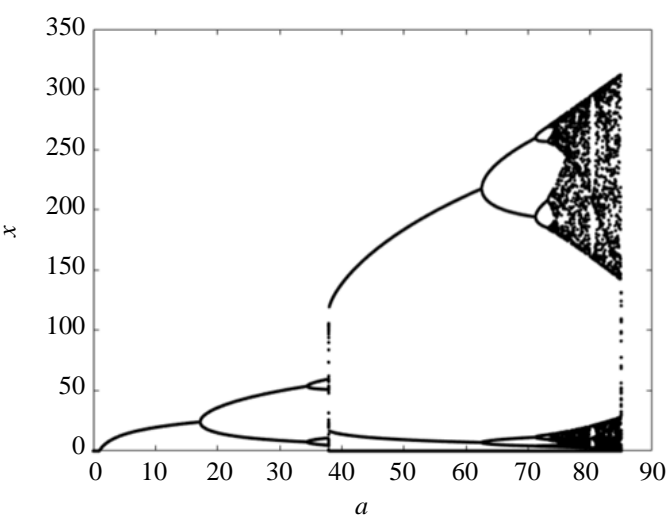

(a)

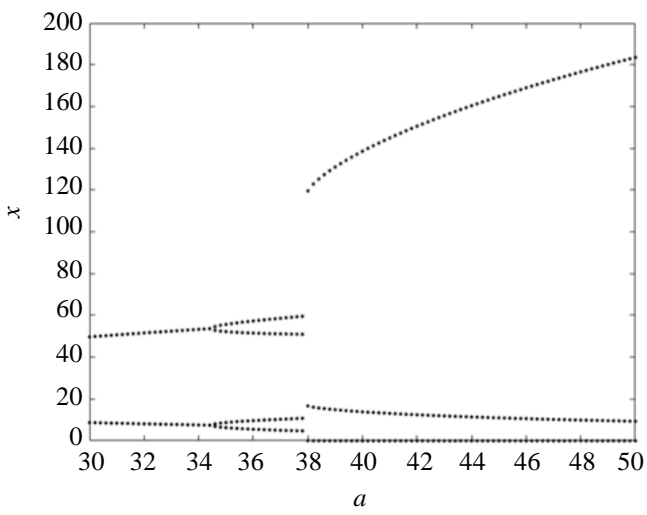

(c)

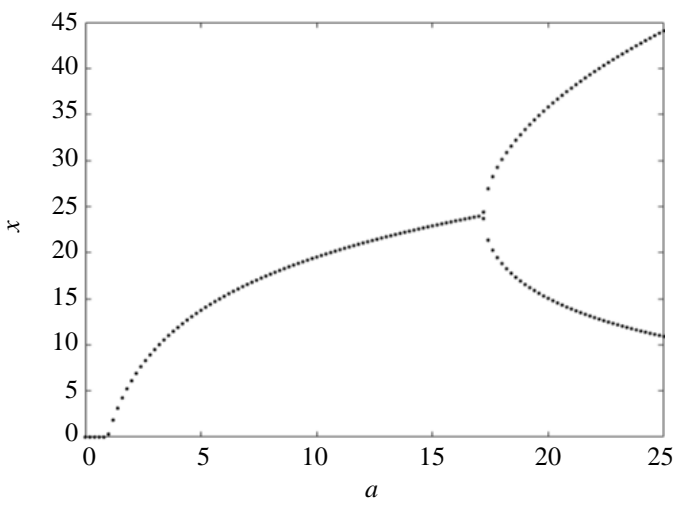

(b)

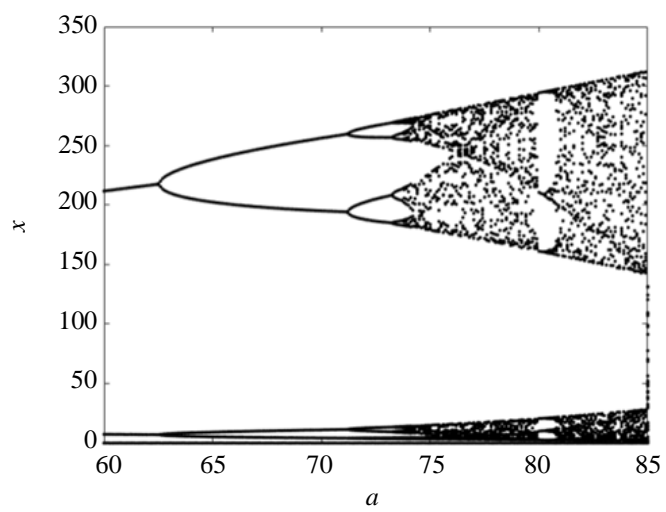

(d)

Fig. 1: Bifurcation diagrams of Ricker mapping (a-d) $(\gamma=0.2, \lambda=0.1, b=0.2,0<a<90)$

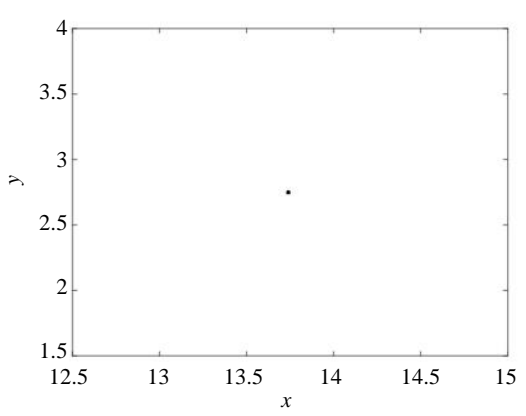

(a)

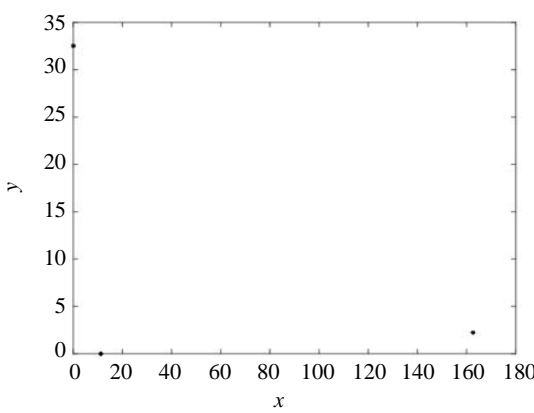

(d)

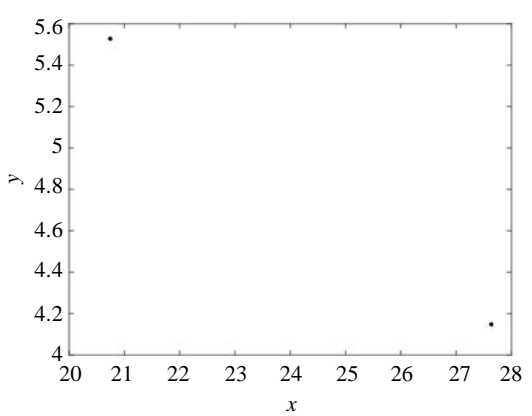

(b)

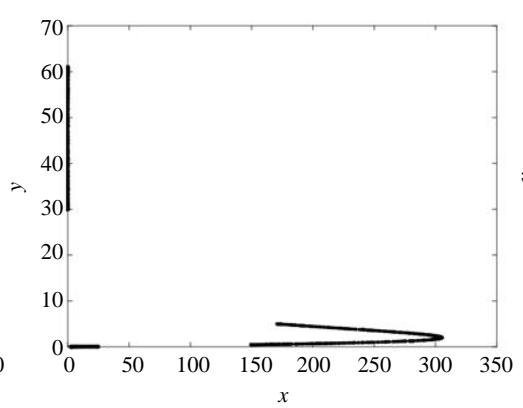

(e)

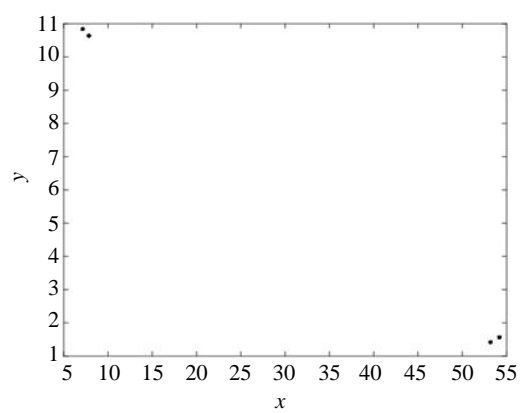

(c)

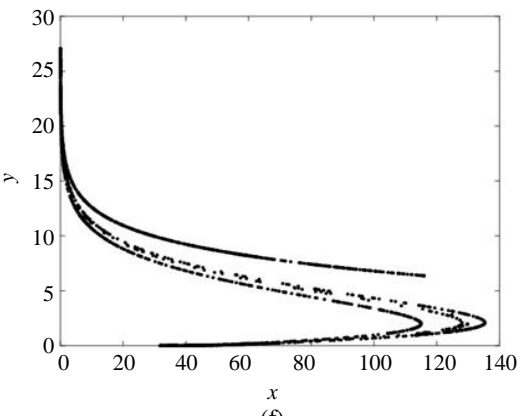

(f)

Fig. 2: The phase diagrams of Ricker mapping ((a). $0.455<a<17.5$, (b). $a=17.5$, (c). $a=34.5$, (d). $34.5<a<60$, (e). $60<a<85$, (f). $a=$ 85) 


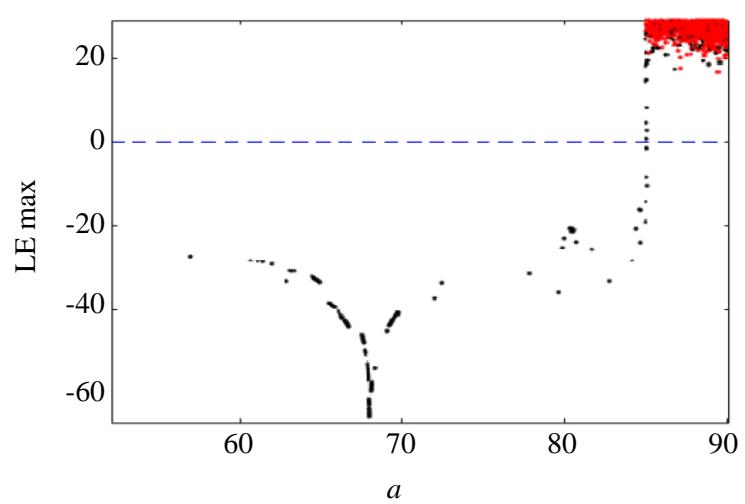

Fig. 3: Lyapunov exponents of Ricker mapping $(\gamma=0.2, \lambda=$ $0.1, b=0.2,0<a<90)$.

\section{Pole Placement Method}

The system is written as a discrete form (Romeiras et al., 1992):

$$
Z_{i+1}=F\left(Z_{i}, a\right), Z_{i} \in R^{2}, a \in R
$$

$F$ is soft enough and $a$ is the actual parameter that can be adjusted externally. Through the OGY method, when the state orbit enters the unstable periodic orbit and stabilizes due to the ergonomic nature of the disordered mechanics, the orbit is controlled by applying the feedback control law to move to the required unstable periodic orbit.

If $Z *(\bar{a})$ is not the stable fixed point proposed by Major Taylor. Equation 11 can be written:

$$
\begin{aligned}
& Z_{i+1}-Z_{*}(\bar{a})=\mathrm{A}\left(Z_{i}-Z_{*}(\bar{a})\right)+\mathrm{B}(a-\bar{a}) \\
& a-\bar{a}=-\mathrm{K}^{\mathrm{T}}\left(Z_{i}-Z_{*}(\bar{a})\right)
\end{aligned}
$$

Replace Equation (13) into Equation (12):

$$
Z_{i+1}-Z_{*}(\bar{a})=\left(\mathrm{A}-\mathrm{BK}^{\mathrm{T}}\right)\left(Z_{i}-Z_{*}(\bar{a})\right)
$$

So as long as the matrix $A-B K^{T}$ is almost stable. In other words, if the coefficient of the eigenvalue is no more than 1 , that fixed spot $Z *(\bar{a})$ is stable:

$$
W=\left(\begin{array}{ccccc}
a_{n-1} & a_{n-2} & \ldots & a_{1} & 1 \\
a_{n-2} & a_{n-3} & \ldots & 1 & 0 \\
\ldots & \ldots & \ldots & \ldots & \ldots \\
a_{1} & 1 & \ldots & 0 & 0 \\
1 & 0 & \ldots & 0 & 0
\end{array}\right)
$$

$a_{i}(i=1, \ldots, n)$ which is the matrix characteristic polynomial coefficients $A$ :

$$
\operatorname{det}(\lambda \mathrm{I}-\mathrm{A})=\lambda^{n}+a_{1} \lambda^{n-1}+\cdots+a_{n}
$$

And $\alpha_{1}, \alpha_{2}, \ldots \alpha_{n}$, are characteristic polynomial coefficients of the characteristic polynomial $\operatorname{det}\left(A-B K^{T}\right)$ :

$\operatorname{det}\left(s \mathrm{I}-\left(\mathrm{A}-\mathrm{BK}^{\mathrm{T}}\right)\right)=s^{n}+\alpha_{1} s^{n-1}+\cdots+\alpha_{n}$

After working out $K^{T},\left|K^{T}\left(Z_{i}-Z *(\bar{a})\right)\right|<\delta$ is obtained by $|a-\bar{a}|<\delta$ and Equation (14).

\section{The Chaos Control of Ricker Mapping}

As shown in Fig. 4 , at $\gamma=0.2, \lambda=0.1, b=0.2$, when $a=85$, there are 1 and 2 points in the mechanics. The dynamic behavior of it is confusing. In the meantime, a strange attractor closed the saddle point unstable manifold. Strange attractors have an infinite number of unstable periodic tracks. There are unstable fixed points.

There are 1 and 2 points in the mechanics and its dynamic behavior is confusing when $\bar{a}=85$. In the mean time, a strange attractor closed the saddle point unstable manifold.

$\left(x *, y_{*}\right)=(57.07,8.256)$ and the points with period-2 $\left(x_{1}, y_{1}\right)=(63.725,6.03), \quad\left(x_{2}, y_{2}\right)=(101.52,4.57)$ embedded in the strange attractor.

\section{To Control the Period-1 Point of Ricker Mapping}

The control parameter $a$ be a variable near the rated value $\bar{a}=85$, at $\gamma=0.2, b=0.2, \lambda=0.1$, then the matrixes $A$ and $B$ have the following results:

$$
\begin{aligned}
& \mathrm{A}=\left.\left(\begin{array}{cc}
(a-\lambda a x-\lambda \gamma a y) \mathrm{e}^{-\lambda(x+y)} & (\gamma a-\lambda a x-\lambda \gamma a y) \mathrm{e}^{-\lambda(x+y)} \\
b & 0
\end{array}\right)\right|_{\left(x_{*}, y_{*}\right)=(57.07,8.256)}=\left(\begin{array}{cc}
-2.7734 & -3.5426 \\
0.2 & 0
\end{array}\right) \\
& \mathrm{B}=\left.\left(\begin{array}{c}
\left((x+y) \mathrm{e}^{-\lambda(x+y)}\right) \\
0
\end{array}\right)\right|_{\left(x_{*}, y_{*}\right)=(57.07,8.256)}=\left(\begin{array}{c}
0.4394 \\
0
\end{array}\right)
\end{aligned}
$$




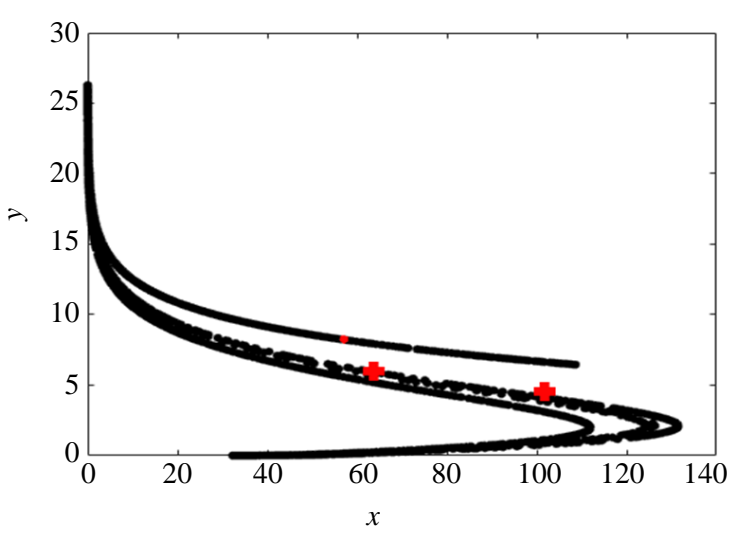

Fig. 4: The strange attractor of the Ricker mapping.

The controllable matrix is a 2-level matrix:

$C=\left(\begin{array}{ll}B & A B\end{array}\right)=\left(\begin{array}{cc}0.4394 & -1.2186 \\ 0 & 0.0879\end{array}\right)$

Then $K^{T}=\left(\alpha_{2}-\alpha_{2}, \alpha_{1}-\alpha_{1}\right) T^{-1}$, where $T=C W$, $W=\left(\begin{array}{cc}a_{1} & 1 \\ 1 & 0\end{array}\right) \cdot a_{i}(i=1,2)$ are the coefficients of $A$ :

$\operatorname{det}(\lambda I-A)=\operatorname{det}\left(\begin{array}{cc}\lambda+2.7734 & 3.5426 \\ -0.2 & \lambda\end{array}\right)$

$=\lambda^{2}+2.7734 \lambda+0.7085$

So $a_{1}=2.7734, a_{2}=0.7085$ :

$T=C W=\left(\begin{array}{cc}0.4394 & -1.2186 \\ 0 & 0.0879\end{array}\right)\left(\begin{array}{cc}2.7734 & 1 \\ 1 & 0\end{array}\right)$

$=\left(\begin{array}{cc}2.1612 & 0.4394 \\ 0.0879 & 0\end{array}\right)$

thus:

$$
T^{-1}=\left(\begin{array}{cc}
0 & 11.3766 \\
2.2758 & -55.9559
\end{array}\right)
$$

The characteristic part of A could be obtained from a fixed point as well $\left(x_{*}, y_{*}\right)=(57.07,8.256)$ are $\lambda_{s}=$ 0.2847, $\lambda_{u}=-2.4887$. The coefficient of $A-B K^{T}$ are $\alpha_{1}, \alpha_{2}$ are. Suppose characteristic roots $\mu_{1}, \mu_{2}$ are named the adjusted value, that is:

$$
\operatorname{det}\left(s I-\left(A-B K^{T}\right)\right)=s^{2}-\left(\mu_{1}+\mu_{2}\right) s+\mu_{1} \mu_{2}
$$

The relationship between the root and the coefficient is as follows:

$\alpha_{1}=-\left(\mu_{1}+\mu_{2}\right), \alpha_{2}=\mu_{1} \mu_{2}$
When $\mu_{1}=1, \alpha_{1}=-1-\alpha_{2}$; when $\mu_{1}=-1, \alpha_{1}=1+\alpha_{2}$ and when $\mu_{1} \mu_{2}=1, \alpha_{2}=1$.

From the previous introduction, we know the matrix $K^{T}$ is not unique. When the matrix $K^{T}$ is obtained with the values $\alpha_{1}, \alpha_{2}$.

You can make the matrix $A-B K^{T}$ asymptotically stable in the triangular area as shown in Fig. 5. In other words, because the coefficient of the eigenvalue is less than 1 , then you can found: $\mu_{1}=0, \mu_{2}=\lambda_{s}$. As $\left(\alpha_{1}, \alpha_{2}\right)=\left(-\lambda_{s}, 0\right)$, we can obtain:

$$
K^{T}\left(0-a_{2},-\lambda_{s}-a_{1}\right) T^{-1}=(-5.6638,147.3177)
$$

When $K^{T}$ is found, $K^{T},\left|K^{T}\left(Z_{i}-Z *(\bar{a})\right)\right|<\delta$ is obtained by $|a-\bar{a}|<\delta$ and Equation (14). Thus there is a region whose width is $2 \delta /\left|K^{T}\right|$, when $Z_{i}$ Parameters are controlled in this area. If not, the parameters are not controlled. We can get the control rate from this formula:

$a-\bar{a}=-\mathrm{K}^{\mathrm{T}}\left(Z_{i}-Z_{*}(\bar{a})\right)$
$\times u\left(\delta-\left|\mathrm{K}^{\mathrm{T}}\left(Z_{i}-Z_{*}(\bar{a})\right)\right|\right)$

where, $u$ is a step function:

$$
u(\alpha)= \begin{cases}0, & \alpha<0 \\ 1, & \alpha>0\end{cases}
$$

As shown in Fig. 6, when we choose $\alpha_{1}=2.7734, \alpha_{2}$ $=0.7085$ in the triangle region of Fig. 5 , in Fig. $6 \mathrm{a}$ and $6 \mathrm{c}$ we can control chaotic movements in cycle 1 orbit at $n=2430$. When we choose $\alpha_{1}=-0.5, \alpha_{2}=1$, you can control chaotic movements in Cycle 1 orbit at $n=4130$ as in Fig. $6 \mathrm{~b}$ and $6 \mathrm{~d}$. The different value makes the control time different.

\section{Control of Period-2 of Ricker Mapping}

By iterating the Equation (10), we can obtain:

$$
R\left(R_{a, b}(x, y)\right):\left\{\begin{array}{l}
F(x, y, a) \\
G(x, y, a)
\end{array}\right.
$$

where:

$$
\begin{aligned}
& F(x, y, a) \\
& =\left(a^{2}(x+\gamma y)(1+x+y)^{-\beta}+\gamma a y\right)\left(1+(a x+\gamma a y)(1+x+y)^{-\beta}+y\right)^{-\beta} \\
& G(x, y, a)=b^{2} x
\end{aligned}
$$

When $\bar{a}=85$, the points $\left(x_{1}, y_{1}\right)$ and $\left(x_{2}, y_{2}\right)$ of the period two are $(63.725,6.03),(101.52,4.57)$, we can get follows $F(x, y, a)=x, G(x, y, a)=y$. 


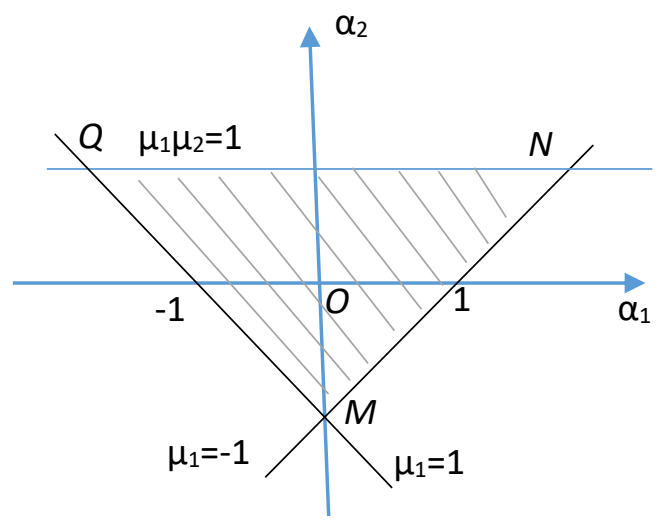

Fig. 5: Choice of regulator poles area

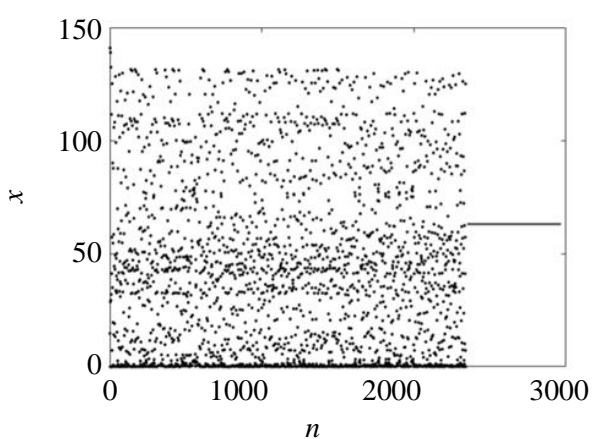

(a)

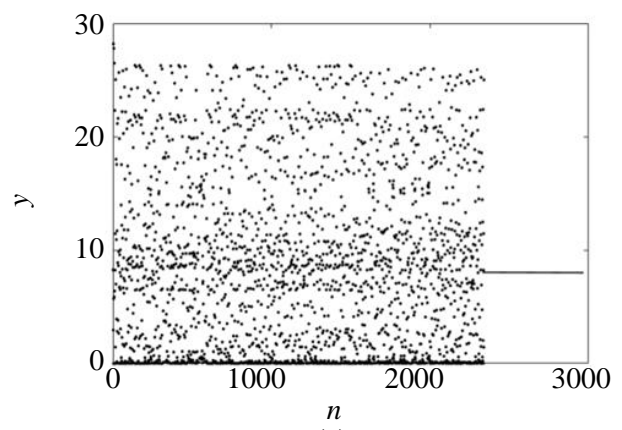

(a)

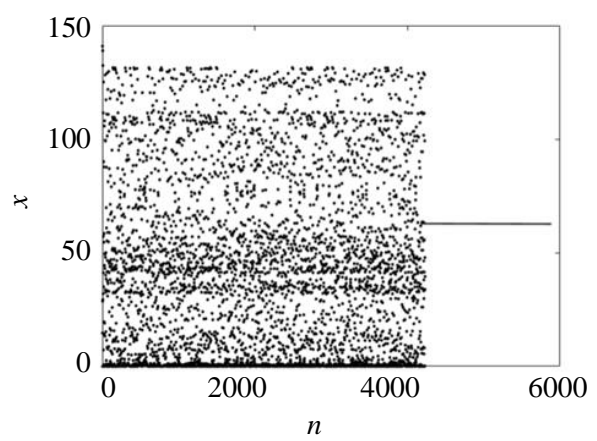

(b)

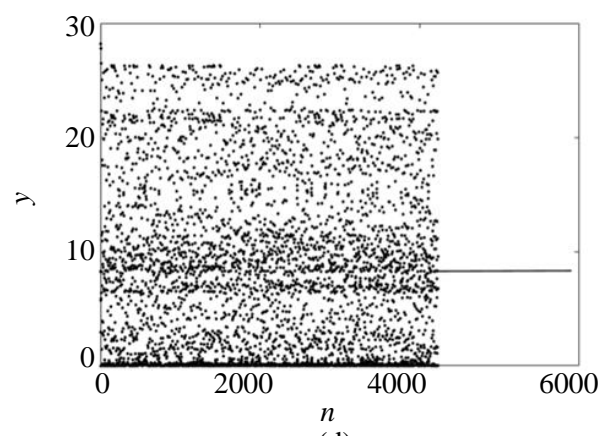

(d)

Fig. 6: Control of period-1 of Ricker mapping

According to:

$$
\begin{aligned}
& A_{1}=\left(\begin{array}{ll}
\partial F(x, y, a) / \partial x & \partial F(x, y, a) / \partial y \\
\partial G(x, y, a) / \partial x & \partial G(x, y, a) / \partial y
\end{array}\right)_{\left(x_{1}, y_{1}\right)} \\
& A_{2}=\left(\begin{array}{ll}
\partial F(x, y, a) / \partial x & \partial F(x, y, a) / \partial y \\
\partial G(x, y, a) / \partial x & \partial G(x, y, a) / \partial y
\end{array}\right)_{\left(x_{2}, y_{2}\right)}
\end{aligned}
$$

and:

$$
B_{1}=\left(\begin{array}{l}
\partial F(x, y, a) / \partial a \\
\partial G(x, y, a) / \partial a
\end{array}\right)_{\left(x_{1}, y_{1}\right)}
$$

$$
B_{2}=\left(\begin{array}{l}
\partial F(x, y, a) / \partial a \\
\partial G(x, y, a) / \partial a
\end{array}\right)_{\left(x_{2}, y_{2}\right)}
$$

\section{Where:}

$$
\begin{aligned}
& \partial F(x, y, a) / \partial x=\left(a^{2}-\lambda a^{2} x-\lambda a^{2} \gamma y+\left(\lambda a^{2} x+\lambda a^{2} \gamma y-\lambda a\right)\right. \\
& \left.\left(\left(a^{2} x+a^{2} \gamma y\right) \mathrm{e}^{-\lambda(x+y)}+a \gamma y\right)\right) \mathrm{e}^{-\lambda\left((a x+\gamma a y) \mathrm{e}^{-\lambda(x+y)}+y\right)} \mathrm{e}^{-\lambda(x+y)}
\end{aligned}
$$

$$
\begin{aligned}
& \partial F(x, y, a) / \partial y=\left(\begin{array}{l}
\left(a^{2} \gamma-\lambda a^{2} x-\lambda a^{2} \gamma y\right) \mathrm{e}^{-\lambda(x+y)}+a \gamma \\
+\left(\left(\lambda^{2} a x+\lambda^{2} a \gamma y-a \lambda \gamma\right) \mathrm{e}^{-\lambda(x+y)}-\lambda\right)
\end{array}\right. \\
& \left.\left(\left(a^{2} x+a^{2} \gamma y\right) \mathrm{e}^{-\lambda(x+y)}+a \gamma y\right)\right) \mathrm{e}^{-\lambda\left((a x+\gamma a y) \mathrm{e}^{-\lambda(x+y)}+y\right)}
\end{aligned}
$$




$$
\begin{aligned}
& \partial G(x, y, a) / \partial x=b^{2}, \partial G(x, y, a) / \partial y=0 \\
& \partial F(x, y, a) / \partial a \\
& =\left((2 a x+2 a \gamma y) \mathrm{e}^{-\lambda(x+y)}+\gamma y-(\lambda x+\lambda \gamma y) \mathrm{e}^{-\lambda(x+y)}\right. \\
& \left.\left(\left(a^{2} x+a^{2} \gamma y\right) \mathrm{e}^{-\lambda(x+y)}+a \gamma y\right)\right) \mathrm{e}^{-\lambda\left(\left((a x+\gamma a y) \mathrm{e}^{-\lambda(x+y)}+y\right)\right.} \\
& \partial G(x, y, a) / \partial a=0
\end{aligned}
$$

Bring $\left(x_{1}, y_{1}\right)=(63.725,6.03)$ and $\left(x_{2}, y_{2}\right)=(101.52$, 4.57) into the Equation (34-38):

$$
\begin{aligned}
& A_{1}=\left(\begin{array}{cc}
-2.54 & -2.98 \\
0 & -4.23
\end{array}\right), A_{1}=\left(\begin{array}{cc}
0.065 & 0.98 \\
0 & -3.27
\end{array}\right) \\
& B_{1}=\left(\begin{array}{c}
0 \\
0.45
\end{array}\right), B_{1}=\left(\begin{array}{c}
0 \\
-0.72
\end{array}\right)
\end{aligned}
$$

Thus the controllable matrices are obtained as following:

$$
C_{1}=\left(\begin{array}{ll}
B_{1} & A_{1} B_{1}
\end{array}\right)=\left(\begin{array}{cc}
0 & -1.34 \\
0.45 & -1.90
\end{array}\right)
$$

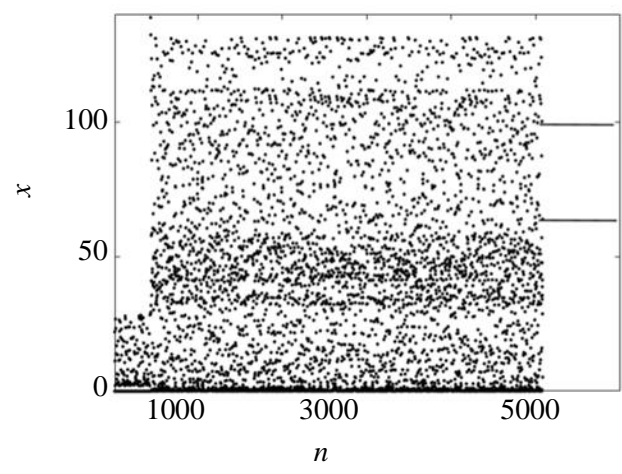

(a)

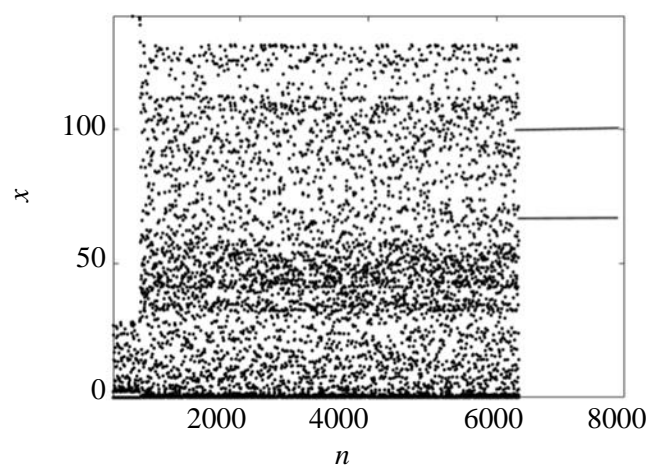

(c)

$$
C_{2}=\left(\begin{array}{ll}
B_{2} & A_{2} B_{2}
\end{array}\right)=\left(\begin{array}{cc}
0 & 0.44 \\
-0.72 & 6.23
\end{array}\right)
$$

$a_{i}(i=1,2)$ are the coefficients of $A_{1}, a_{1}=4.23, a_{2}=$ 1074 , In the mean time the characteristic roots are $\lambda_{1 s}=$ $-0.58, \lambda_{1 u}=-4.45$. The solution to the pole assignment problem is as follows:

$K_{1}^{T}=\left(\alpha_{2}-a_{2}, \alpha_{1}-a_{1}\right) T_{1}^{-1}$

According to the discussion of the values $a_{1}, a_{2}$, we still take $\left(a_{1}, a_{2}\right)=\left(-\lambda_{1 s}, 0\right)$, where $T_{1}=C_{1} W_{1}$, so:

$$
\begin{aligned}
& K_{1}^{T}=\left(0-a_{2},-\lambda_{1 s}-a_{1}\right) T_{1}^{-1} \\
& =(-4.1420,-4.3561)
\end{aligned}
$$

$a_{i}(i=3,4)$ are the coefficients of $A_{2}-B_{2} K_{2}^{T}, a_{3}=$ 2.65, $a_{4}=-0.15$, the characteristic roots is $\lambda_{2 s}=0.043$, $\lambda_{2 u}=-4.12$. We take $\left(a_{1}, a_{2}\right)=\left(-\lambda_{2 s}, 0\right)$, thus we obtain $K_{2}^{T}=(-0.3947,6.1640)$ :

$$
\begin{aligned}
& a-\bar{a}=-\mathrm{K}_{i}^{\mathrm{T}}\left(Z_{n}-Z(i)(\bar{a})\right) \\
& \times u\left(\delta-\left|\mathrm{K}_{i}^{\mathrm{T}}\left(Z_{n}-Z(i)(\bar{a})\right)\right|\right),(i=1,2)
\end{aligned}
$$

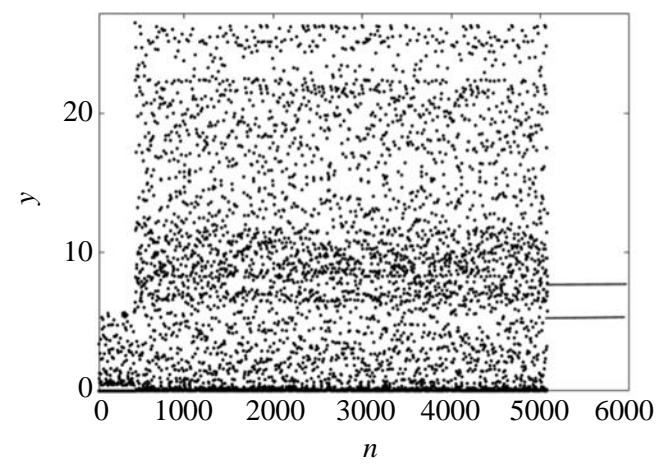

(b)

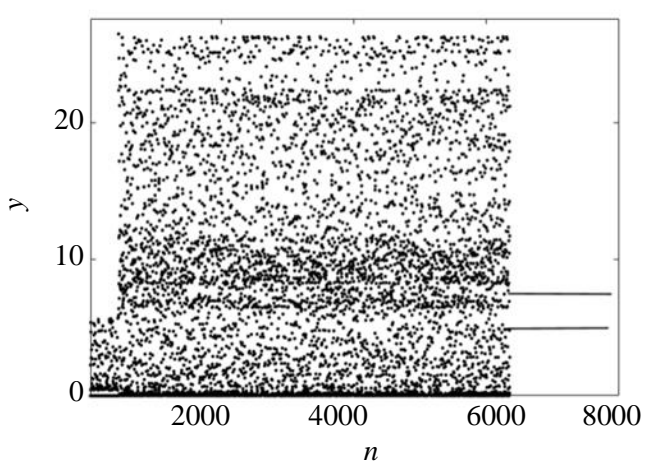

(d)

Fig. 7: The chaotic motion is controlled 
As shown in Fig. 7, when $a_{1}=-0.47, a_{2}=0, a_{3}=-0.28$, $a_{4}=0$ in the chaotic movement of the triangular area in Fig. 5 could be controlled in period- 2 orbits at $n=5080$ in Fig. $7 \mathrm{a}$ and $7 \mathrm{~b}$. When $a_{1}=-0.3, a_{2}=0.2, a_{3}=-0.25, a_{4}=$ 0.54 , we can controlled the chaotic motion on the period-2 orbits at $n=6350$ as Fig. $7 \mathrm{c}$ and $7 \mathrm{~d} 4$.

\section{Conclusion}

The dynamic behavior in the Ricker mapping is very complex in different values of $a$. When the value of $a$ is changed from 0.455 to 90 , the mapping goes through doubling bifurcation to Neimark-Sacker bifurcation. Several strange attractors coexist at last. Through the numerical simulation and analysis of the bifurcation and phase diagrams of the mapping, it is consistent with the theoretical analysis. It controls the chaotic control of Ricker mapping, selects the amount of perturbation of the control parameter as the pole construction method by linear control theory. Compared with other control methods, this control method takes less time, costs the least and has the best control effect. Studies have shown that ecological balance can be achieved by appropriately adjusting the birth rate $a$, survival rate $b$.

\section{Acknowledgment}

This work is supported by the High Level Scientific Research Project Cultivation Fund of Shandong Women's University.

\section{Ethics}

The authors claim that there is no ethical issue.

\section{References}

Berdnikov, V., \& Lokhin, V. (2019). Synthesis of guaranteed stability regions of a nonstationary nonlinear system with a fuzzy controller. Civil Engineering Journal, 5(1), 107-116.

Caswell, H. (2001). Matrix Population Models: Construction, Analysis and Interpretation. 2nd edn Sinauer Associates. Inc., Sunderland, MA.

El-Sayed, A. M. A., Elsaid, A., Nour, H. M., \& Elsonbaty, A. (2013). Dynamical behavior, chaos control and synchronization of a memristor-based ADVP circuit. Communications in Nonlinear Science and Numerical Simulation, 18(1), 148-170.
Feng, G., Pan, J. X., Yong, M., \& Ji, Z. H. E. N. (2018). The dynamic property and chaos control for a two-degree-of-freedom vibro-impact system.

Iu, H. H. C., Yu, D. S., Fitch, A. L., Sreeram, V., \& Chen, H. (2011). Controlling chaos in a memristor based circuit using a twin-T notch filter. IEEE Transactions on Circuits and Systems I: Regular Papers, 58(6), 1337-1344.

Kocarev, L., \& Parlitz, U. (1995). General approach for chaotic synchronization with applications to communication. Physical review letters, 74(25), 5028.

Leslie, P. H. (1945). On the use of matrices in certain population mathematics. Biometrika, 33(3), 183-212.

Leslie, P. H. (1948). Some further notes on the use of matrices in population mathematics. Biometrika, 35(3/4), 213-245.

Nik, H. S., Effati, S., \& Saberi-Nadjafi, J. A. F. A. R. (2015). Ultimate bound sets of a hyperchaotic system and its application in chaos synchronization. Complexity, 20(4), 30-44.

Ogata, K. (1990). Controlling engineering. Prentice Hall, Englewood Cliffs, NJ.

Ott, E., Grebogi, C., \& Yorke, J. A. (1990). Controlling chaos. Physical review letters, 64(11), 1196.

Pyragas, K. (1993). Predictable chaos in slightly perturbed unpredictable chaotic systems. Physics Letters A, 181(3), 203-210.

Reza Ahrabi, A., \& Kobravi, H. R. (2019). A chaos to chaos control approach for controlling the chaotic dynamical systems using Hamilton energy feedback and fuzzy-logic system. Chaos: An Interdisciplinary Journal of Nonlinear Science, 29(7), 073113.

Romeiras, F. J., Grebogi, C., Ott, E., \& Dayawansa, W. P. (1992). Controlling chaotic dynamical systems. Physica D: Nonlinear Phenomena, 58(1-4), 165-192.

Saini, S., \& Saini, J. S. (2014, December). GA optimized time delayed feedback control of chaos in a memristor based chaotic circuit. In 2014 IEEE Symposium on Computational Intelligence for Engineering Solutions (CIES) (pp. 74-80). IEEE.

Ugarcovici, I., \& Weiss, H. (2004). Chaotic dynamics of a nonlinear density dependent population model. Nonlinearity, 17(5), 1689.

Ugarcovici, I., \& Weiss, H. (2007). Chaotic attractors and physical measures for some density dependent Leslie population models. Nonlinearity, 20(12), 2897. 